\title{
Portalez, Christophe: Alfred Naquet et ses amis politiques. Patronage, corruption et scandale en République (1870-1898), 274 S., PUR, Rennes 2018.
}

\author{
Daniel Mollenhauer
}

Online publiziert: 4. Mai 2020

(C) Der/die Autor(en) 2020

In Frankreich ist Alfred Naquet vor allem als der père du divorce, als Vater des modernen Scheidungsrechts bekannt. Dass die Verkürzung auf ein singuläres Ereignis einer über 30 Jahre andauernden politischen Karriere, die vom ausgehenden Kaiserreich bis zur Dreyfus-Affäre reichte, nicht gerecht wird, zeigt Christophe Portalez in der hier vorzustellenden Studie. Denn obwohl er es nie bis zum Ministerrang brachte und obwohl sein Wirken (auch in der Selbstwahrnehmung) vor allem durch Misserfolge gekennzeichnet war, spielte Naquet doch in nahezu allen großen Auseinandersetzungen, die die Gründungsjahrzehnte der Dritten Republik prägten, eine wichtige Rolle.

Portalez' Buch will jedoch keine klassische Politiker-Biografie sein: Wenig ist über die Kindheit und Jugend Naquets zu lesen, wenig über seine Identität als assimilierter, nicht praktizierender Jude. Vielmehr soll die Karriere Naquets dem Autor dazu dienen, exemplarisch die Problemkreise von Patronage und Korruption sowie die daraus resultierenden öffentlichen Skandale in den Gründungsjahren der Dritten Republik zu untersuchen. Die Relevanz dieser Thematik ist nicht zu übersehen, wurde doch das im Krieg 1870/71 installierte neue Regime von Beginn an stets von dem Vorwurf eines ausufernden Klientelismus begleitet.

Naquet bietet sich für eine derartige Arbeit aus mehreren Gründen an: Zum einen, weil er selbst früh begonnen hat, das Patronage- und Klientelsystem zwischen Abgeordneten, lokalen Honoratioren und Wählern zu reflektieren und zu kritisieren; zum zweiten, weil er während seiner Karriere mehrfach in Skandale verwickelt war, die mit Patronage/Nepotismus oder Korruption zu tun hatten. Problematisch ist allerdings die Quellenlage: Zwar war Naquet regelmäßig - wie viele seiner Kollegen - neben seiner parlamentarischen Tätigkeit auch publizistisch aktiv, ein nennens-

D. Mollenhauer $(\bowtie)$

Ludwig-Maximilians-Universität München, München, Deutschland

E-Mail: Daniel.Mollenhauer@1mu.de 
werter Nachlass, eine zusammenhängende Korrespondenz ist jedoch nicht erhalten. Daher verfolgt Portalez sein Thema nicht systematisch, sondern nimmt immer dort Sondierungen vor, wo es die Quellenlage erlaubt. So untersucht er für die 1870er Jahre die besonders umkämpften Wahlen im Département Vaucluse, deren Ergebnisse von der unterlegenen Partei regelmäßig angefochten wurden (Kap. 2-4). Die folgenden parlamentarischen Untersuchungen über die Einflussnahme von Beamten, Einschüchterungsversuche oder Wahlversprechungen erlauben einen Blick hinter die Kulissen der offiziellen, sichtbaren Wahlkämpfe, der sonst nur schwer möglich gewesen wäre. Einblicke in das Verhältnis von Abgeordneten und Bürgern in solchen ,Normalzeiten“ gewinnt Portalez durch eine Untersuchung der Empfehlungsschreiben, die Naquet und seine Kollegen im Département Vaucluse für Bürger ihres jeweiligen Wahlkreises verfassten, die sich um eine Stelle oder eine Beförderung in der Post- und Telegrafenverwaltung bemühten (Kap. 5). Die recommandations waren einerseits ein Instrument der Abgeordneten, sich eine Klientel zu schaffen und im Wahlkreis als einflussreich und daher ,nützlich` zu gelten, andererseits dienten sie dazu, die Verwaltung (gerade das Amt des Briefträgers galt als politisch sensibel) zu republikanisieren.

Naquet sah diese auch von ihm selbst regelmäßig praktizierten Interventionen ausgesprochen kritisch: Sowohl in seinem privaten Briefwechsel mit dem ehemaligen General der Pariser Commune Georges Cluseret als auch in seinem programmatischen Buch „Questions constitutionelles“ entfaltete er eine scharfe Kritik an dem Patronage- und Klientelsystem, das er für eine Pervertierung des Parlamentarismus verantwortlich machte. Denn die Empfehlungen raubten den Abgeordneten nicht nur einen Großteil ihrer Arbeitszeit, sondern sie brachten auch ihre Unabhängigkeit in Gefahr: Nur derjenige Abgeordnete, der das Ohr des Ministers hatte, konnte erfolgreich Empfehlungen aussprechen. Wenn ihm seine Wiederwahl am Herzen lag, war er also gut beraten, seinen Oppositionsgeist zu zügeln, auch wenn dies seinen politischen Überzeugungen widersprach und die Kontrollfunktion des Parlaments letztlich aushebelte (Kap. 6).

Naquets Bemühungen, durch eine Revision der Verfassung diese Fehlentwicklungen zu korrigieren, sind die beiden folgenden Kapitel gewidmet. Überzeugend zeigt Portalez, wie Naquets wachsende Kritik an der sich herauskristallisierenden Verfassungswirklichkeit der Dritten Republik ihn schließlich in den Boulangismus, die bizarr anmutende Allianz zwischen radikalen Republikanern, Nationalisten, Bonapartisten und katholischen Monarchisten, führte - eine Allianz, die ihn von seinen bisherigen Parteifreunden entfremdete und ihn zu einem parlamentarischen Einzelgänger werden ließ.

Dass die Unabhängigkeit des Abgeordneten nicht nur durch die Klientelbeziehungen zwischen ihm und seinen Wählern, sondern auch durch die wirtschaftlichen Verflechtungen zwischen Politik und Unternehmen gefährdet werden können, thematisiert Portalez abschließend anhand der Interessen, die Naquet mit der Société génerale pour la fabrication de la dynamite verbanden. Persönliche, familiäre und wissenschaftliche Beziehungen bildeten den Ausgangspunkt für diese Verbindung, die auch nach dem politischen Erfolg Naquets nicht abriss. Politisch relevant wurden sie erst im Zuge des Panamaskandals, dessen parlamentarische und gerichtliche 
Aufarbeitung einen detaillierten Einblick in das Zusammenwirken von politischen und wirtschaftlichen Interessen erlaubt.

Das innovative Potenzial der Studie ist groß: Denn der Frage, wie Politik in Frankreich vor dem Entstehen der modernen, strukturierten und bürokratisierten Parteien eigentlich funktionierte, ist von der Forschung bislang deutlich zu wenig Aufmerksamkeit geschenkt worden. Erfreulich ist auch, dass Portalez systematisch die vielfältigen Forschungen zu Klientel- und Netzwerkbildung, Korruption und Skandal sowohl aus anderen Epochen (insbesondere der Frühen Neuzeit) als auch aus anderen politischen Kulturen in seine Überlegungen einbezieht. Ganz überzeugen kann das Buch dennoch nicht. Leider bleiben die ,Freunde“ Naquets, also sein Netzwerk aus Klienten und Kollegen, relativ blass und konturlos. Dies ist zum einen der Tatsache geschuldet, dass der Autor seine Themen jeweils nur punktuell, nicht aber über einen längeren Zeitraum untersucht: Wie funktionierten die Wahlkämpfe im Departement, nachdem sich die Republik in den 1880er Jahren konsolidieren konnte? Wie verhielt es sich mit Naquets Netzwerken in Carpentras/Vaucluse, als er 1893 dort erneut als Abgeordneter kandidiert und sogar gewählt wurde? Auf welche ,Freunde' konnte er sich nach dem boulangistischen Abenteuer noch oder wieder verlassen? Zum anderen liegt es daran, dass Portalez sich nicht recht entscheiden kann, ob er auf Naquet als Hauptprotagonisten oder auf die Gruppe der Republikaner des Vaucluse fokussieren soll. Dies erklärt wohl auch, weshalb sich eingestreut immer wieder Passagen finden, die eher biografischen Charakter haben und in denen der thematische Fokus aus dem Blick gerät. Dennoch: Eine lesenswerte Studie, die wichtige Erkenntnisse über Praxis und Wahrnehmung von Klientelismus und Korruption in der frühen Dritten Republik vermittelt.

Funding Open Access funding provided by Projekt DEAL.

Open Access Dieser Artikel wird unter der Creative Commons Namensnennung 4.0 International Lizenz veröffentlicht, welche die Nutzung, Vervielfältigung, Bearbeitung, Verbreitung und Wiedergabe in jeglichem Medium und Format erlaubt, sofern Sie den/die ursprünglichen Autor(en) und die Quelle ordnungsgemäß nennen, einen Link zur Creative Commons Lizenz beifügen und angeben, ob Änderungen vorgenommen wurden.

Die in diesem Artikel enthaltenen Bilder und sonstiges Drittmaterial unterliegen ebenfalls der genannten Creative Commons Lizenz, sofern sich aus der Abbildungslegende nichts anderes ergibt. Sofern das betreffende Material nicht unter der genannten Creative Commons Lizenz steht und die betreffende Handlung nicht nach gesetzlichen Vorschriften erlaubt ist, ist für die oben aufgeführten Weiterverwendungen des Materials die Einwilligung des jeweiligen Rechteinhabers einzuholen.

Weitere Details zur Lizenz entnehmen Sie bitte der Lizenzinformation auf http://creativecommons.org/ licenses/by/4.0/deed.de. 